\title{
Multinuclear Magnetic Resonance Relaxation Studies of Binary Solutions of Pivalic Acid and Carbon Tetrachloride in Liquid and Solid Phases
}

\author{
Dagfinn W. Aksnes*,a and Liudvikas L. Kimtys ${ }^{b}$ \\ a Department of Chemistry, University of Bergen, N-5007 Bergen, Norway and bepartment of Physics, Vilnius \\ University, Universiteto Str. 3, Vilnius 2734, Lithuania
}

\begin{abstract}
Aksnes, D. W. and Kimtys, L. L., 1993. Multinuclear Magnetic Resonance Relaxation Studies of Binary Solutions of Pivalic Acid and Carbon Tetrachloride in Liquid and Solid Phases. - Acta Chem. Scand. 47: 27-32.

The binary system pivalic acid-carbon tetrachloride was studied in the liquid and solid phases using multinuclear NMR. The courses of the melting and transition point lines as a function of composition and temperature were determined. The ${ }^{1} \mathrm{H}$ linewidth measurements indicate that translational diffusion in the disordered phase is occurring at a much faster rate in the dilute samples. The corresponding activation energy decreases monotonically from $59 \mathrm{~kJ} \mathrm{~mol}^{-1}$ in neat pivalic acid (PA) to $26 \mathrm{~kJ} \mathrm{~mol}^{-1}$ in the most dilute sample (6\% PA). Two striking features distinguish the ${ }^{1} \mathrm{H}$ spin-lattice relaxation time $\left(T_{1}\right)$ curves in the ordered phase. Firstly, the $T_{1}$ curves broaden on increasing dilution, and secondly, the value of $T_{1}$ at the minimum increases. This indicates that the $T_{1}$ relaxation is described by a distribution of correlation times. The position of the $T_{1}$ minimum also moves to lower temperatures on increasing dilution, implying that the reorientational freedom of the PA molecules is increased by the addition of carbon tetrachloride. The $T_{1}$ data of the carboxyl deuteron reflect the overall tumbling motion. The corresponding activation energy in the liquid phase decreases monotonically from $20 \mathrm{~kJ} \mathrm{~mol}^{-1}$ in neat PA to $8.6 \mathrm{~kJ} \mathrm{~mol}^{-1}$ in the most dilute sample (9\% PA). In the disordered phase the positive slopes of the ${ }^{2} \mathrm{H} T_{1}$ curves imply that the correlation time of the overall motion is longer than $1.6 \mu \mathrm{s}$. This observation is consistent with the high values of the corresponding activation energy $\left(26-30 \mathrm{~kJ} \mathrm{~mol}^{-1}\right)$.
\end{abstract}

Orientational disorder and dynamics in one-component organic crystals have been extensively studied using various techniques. ${ }^{1}$ Three types of motional processes have been consistently found in these investigations. In the ordered phase anisotropic molecular motions occur, whereas in the disordered plastic and liquid phases overall molecular tumbling and translational diffusion take place.

In addition to investigations of one-component disordered crystals it is also of interest to study binary systems where one or both of the components possess disordered phases. However, only very few experimental investigations on such systems have been performed to date. ${ }^{2-4}$ In this paper we therefore report the results of multinuclear NMR studies on the system pivalic acid-carbon tetrachloride. Pivalic acid (PA) was chosen as one of the components (solute) because its phase behaviour and dynamics have been well characterized. ${ }^{1,2,5}$ Carbon tetrachloride, consisting of spherical and non-polar molecules, was chosen as the second component (solvent) to minimize intermolecular forces and to avoid unwanted NMR resonances. Unfortunately, the phase diagram of the $\mathrm{PA}-\mathrm{CCl}_{4}$ system does not seem to have been studied.

\footnotetext{
* To whom correspondence should be addressed.
}

The neat PA molecules form non-polar dimers in the liquid as well as solid phases as shown in Fig. 1.,7 However, although the elongated PA dimers are less symmetrical than the globular $\mathrm{CCl}_{4}$ molecules both compounds exhibit disordered cubic phases. PA melts at $310 \mathrm{~K}$ and undergoes a solid-solid phase transition at $280 \mathrm{~K}^{8}{ }^{8} \mathrm{X}$-Ray studies ${ }^{7,9,10}$ have established that the hightemperature disordered phase (solid I) is face-centred cubic, whereas the low-temperature phase (solid II) is triclinic.

X-Ray diffraction studies ${ }^{11,12}$ and contemporary thermal measurements ${ }^{13,14}$ on carbon tetrachloride revealed that this substance exhibits unusual crystallographic behaviour. It was found that, on cooling from the melt, a face-centred cubic phase (solid Ia) was formed at $245 \mathrm{~K}$ which transformed to a rhombohedral phase (solid Ib) at $234 \mathrm{~K}$, followed by a transformation to a monoclinic phase (solid II) at $217-225 \mathrm{~K}$. When the monoclinic phase was warmed, the rhombohedral phase was again formed; this phase persisted until the melting point (248-250 K).

NMR spectroscopy has provided a wealth of information on phase transitions and reorientational and translational motions occurring in the different phases of one-component molecular solids. ${ }^{1,2}$ The corresponding 
<smiles></smiles>

Fig. 1. The cyclic dimer of PA. The average structure of the dimeric unit is of $C_{2 n}$ symmetry when the hydrogen-bond exchange is slow.

binary systems have, however, received very limited NMR attention. ${ }^{2-4}$ This multinuclear NMR investigation was therefore undertaken to study the phase behaviour and dynamics of the binary system $\mathrm{PA}-\mathrm{CCl}_{4}$ using NMR linewidth and spin-lattice relaxation time measurements.

\section{Experimental}

Pivalic acid (PA) was obtained from Fluka ( $>99.5 \%$ ), and deuterated pivalic acid (PA-d) was prepared from pivalyl chloride and deuterium oxide slightly enriched in ${ }^{17} \mathrm{O}$ (ca. $1.5 \%$ ). The extent of deuteration was measured to be ca. $98 \%$ by ${ }^{1} \mathrm{H}$ NMR spectroscopy. The investigated samples of PA and PA-d were carefully purified using azeotropic distillation and sublimation as explained elsewhere. ${ }^{5}$ Carbon tetrachloride, obtained from RiedeldeHaën $(\geqslant 99.8 \%)$, was used without further purification. Weighed amounts of PA (or PA-d) and $\mathrm{CCl}_{4}$ were transferred into 5 and $10 \mathrm{~mm}$ NMR tubes, degassed by several freeze-pump-thaw cycles and sealed under vacuum.

The ${ }^{1} \mathrm{H},{ }^{2} \mathrm{H}$ and ${ }^{17} \mathrm{O}$ NMR spectra were measured without an internal NMR lock, at 400.13, 61.43 and $54.24 \mathrm{MHz}$, respectively, on a Bruker AM $400 \mathrm{WB}$ spectrometer. The $5 \mathrm{~mm}$ NMR tubes were used for the ${ }^{1} \mathrm{H}$ measurements, whereas the ${ }^{2} \mathrm{H}$ and ${ }^{17} \mathrm{O}$ measurements were carried out using the $10 \mathrm{~mm}$ NMR tubes. ${ }^{1} \mathrm{H}$ composite pulse decoupling (CPD) with a Waltz-16 sequence, covering a range of ca. $8 \mathrm{kHz}$, was used when measuring the ${ }^{2} \mathrm{H}$ NMR spectra. The sample temperature was regulated and stabilized to within $\pm 1 \mathrm{~K}$ by means of a Bruker B-VT 1000 temperature-control unit.

The $T_{1}$ times were measured by standard inversionrecovery $\left(180^{\circ}-\tau-90^{\circ}-T\right)_{n}$, typically using 16 values of $\tau$ and the recycle delay $T \geqslant 5 T_{1} .{ }^{15}$ The ${ }^{1} \mathrm{H},{ }^{2} \mathrm{H}$ and ${ }^{17} \mathrm{O} 90^{\circ}$ pulse lengths were ca. 8,15 and $17 \mu$ s, respectively. Exponential weighting, with a line-broadening factor of $0.5-5 \mathrm{~Hz}$, was applied to the free induction decays. A nonlinear three-parameter fitting of peak heights (including the equilibrium value) was used for calculating the $T_{1}$ values. The reproducibility of $T_{1}$ was within $2 \%$, whereas the real errors are expected to be within $5 \%$.

\section{Results and discussion}

\section{NMR linewidths}

The NMR linewidth $\left(\Delta v_{1 / 2}\right)$ generally undergoes a discontinuous change in connection with a change of state or phase. The courses of the melting and transition point lines as a function of temperature and mole fraction of PA $\left(X_{\mathrm{PA}}\right)$ shown in Fig. 2 were therefore monitored by observing the ${ }^{1} \mathrm{H}$ NMR linewidth. The temperature of the melting point and rotational transition point of PA is lowered by the presence of the other component until a mole fraction of PA of 0.17 is reached. Since the melting occurred over a small temperature range, it was difficult to distinguish between the solidus and liquidus lines on the basis of linewidth measurements. However, the two lines appeared to be roughly coincident in the mixtures studied. A more detailed determination of the phase diagram of the $\mathrm{PA}-\mathrm{CCl}_{4}$ system would require extensive thermodynamic measurements far beyond the scope of this investigation.

On freezing the sample narrow, liquid-like signals appeared on top of the main relatively broad ${ }^{1} \mathrm{H}$ and ${ }^{2} \mathrm{H}$ resonances. The amount of the narrow-line component decreased rapidly when the temperature was lowered. Fig. 3 shows that the amount of the narrow-signal component was negligible after about $4 \mathrm{~h}$ at $280 \mathrm{~K}$. However, to make the experimental work practicable it is essential that the time for equilibration is as short as possible. Fig. 3 demonstrates that the linewidth (converted into $T_{2}$ ) and $T_{1}$ of the broad-line component were independent of the presence of a small amount of the narrow-line component. After the temperature of the sample had been altered it was thus enough to allow $30 \mathrm{~min}$ for equilibration before the measurements were started.

The narrow ${ }^{1} \mathrm{H}$ and ${ }^{2} \mathrm{H}$ resonances observed for solid I (3-10 and 9-34 Hz, respectively, when $X_{\mathrm{PA}} \leqslant 0.7$ ) suggest that this phase is disordered and nearly cubic. For higher PA concentrations, however, the $\mathrm{COO}^{2} \mathrm{H}$ linewidth becomes larger than $1 \mathrm{kHz}$, whereas the ${ }^{1} \mathrm{H}$ linewidths show no increase. The line-broadening of the ${ }^{1} \mathrm{H}$ and ${ }^{2} \mathrm{H}$ signals originates from intermolecular dipole-dipole interactions and intramolecular electric quadrupolar

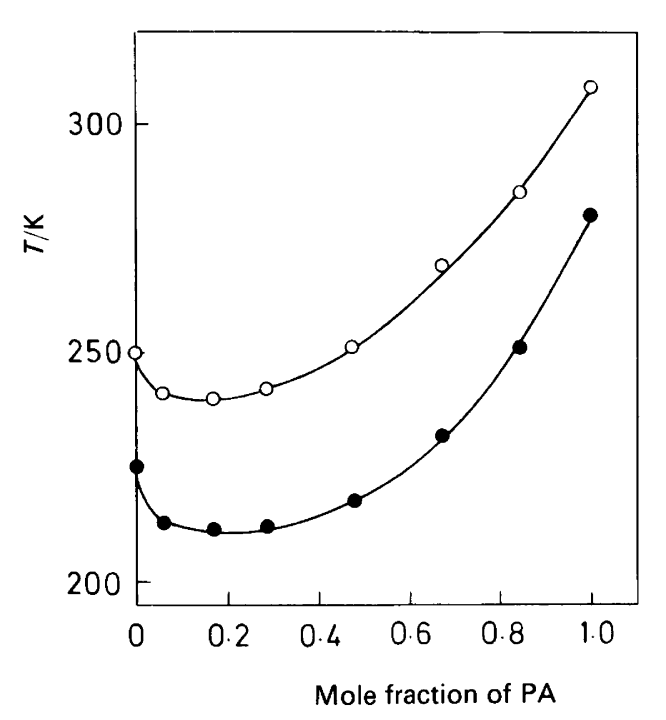

Fig. 2. The melting and transition points of the binary system $\mathrm{PA}-\mathrm{CCl}_{4}$ as a function of composition. 


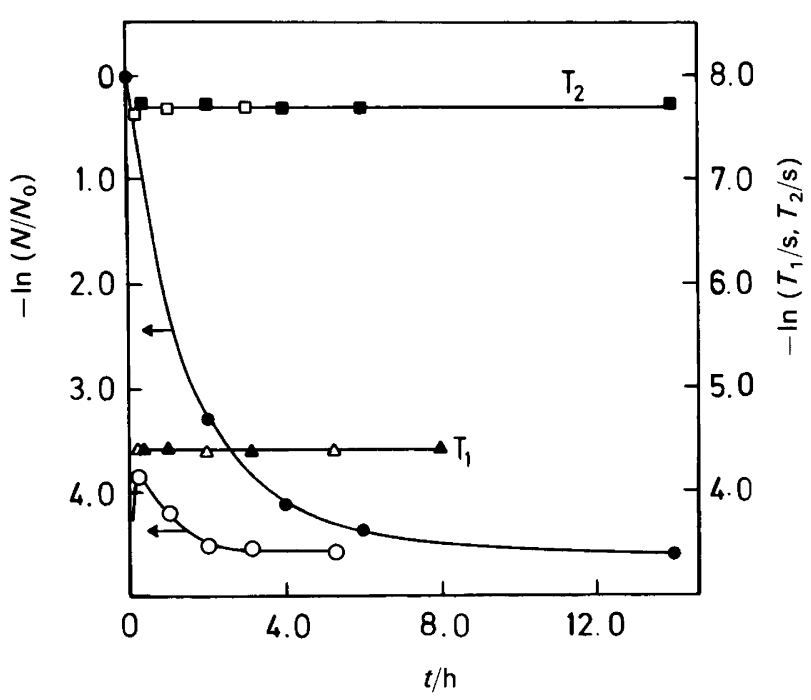

Fig. 3. ${ }^{2} \mathrm{H}$ relaxation times $\left(T_{1}, T_{2}\right)$ and relative contribution of the narrow-line component $\left(N / N_{0}\right)$ as a function of equilibration time at $280 \mathrm{~K}$. All measurements were made on the $\mathrm{COO}^{2} \mathrm{H}$ deuterons of the binary system PA-CCl ${ }_{4}$ at a PA mole fraction of 0.89 by cooling (open symbols) or warming (filled symbols) the sample.

interactions, respectively. The line-narrowing observed in the disordered phase is thus caused by molecular translation and reorientation, respectively. The extensive broadening of the $\mathrm{COO}^{2} \mathrm{H}$ line when $X_{\mathrm{PA}}>0.7$ thus shows that the rate of overall tumbling is significantly reduced.

The width of the ${ }^{1} \mathrm{H}$ resonances, in particular the methyl line, is drastically reduced in all binary samples compared with neat PA. This observation suggests that the translational diffusion is occurring at a much faster rate in the diluted samples. In fact, as shown in Fig. 4,

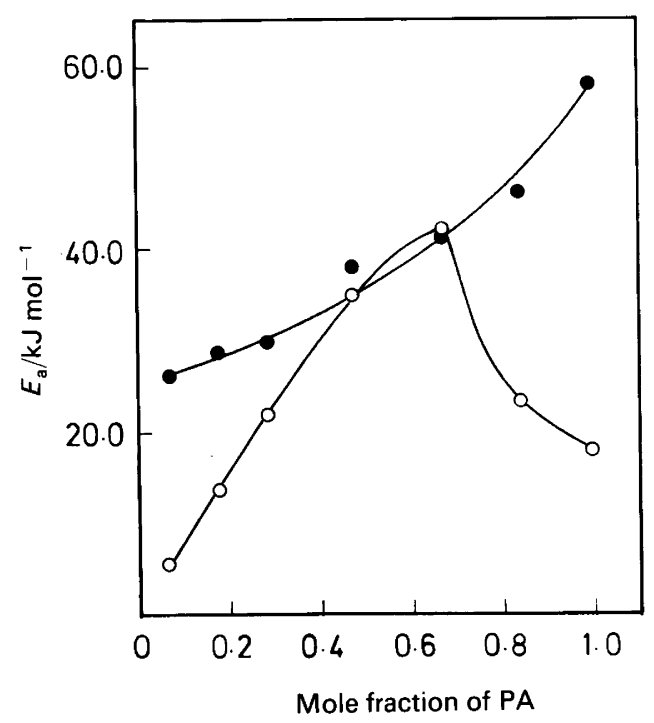

Fig. 4. Activation energy obtained from the linewidths of the $\mathrm{CH}_{3}(\bullet)$ and $\mathrm{COOH}(\mathrm{O})$ protons as a function of composition in the disordered phase of the binary system $\mathrm{PA}-\mathrm{CCl}_{4}$. the activation energy for translational diffusion $\left(E_{\mathrm{d}}\right)$ decreases monotonically for $\mathrm{CH}_{3}$ from $59 \mathrm{~kJ} \mathrm{~mol}^{-1}$ in neat PA $\left(X_{\mathrm{PA}}=1\right)$ to $26 \mathrm{~kJ} \mathrm{~mol}^{-1}$ in the most dilute sample $\left(X_{\mathrm{PA}}=0.06\right)$. It seems that the principal effect of adding $\mathrm{CCl}_{4}$ to $\mathrm{PA}$ is to generate an excessive concentration of line defects in the solid. ${ }^{3,16}$ The defects may provide paths along which the molecules can diffuse more rapidly and with lower activation energy than through the unperturbed lattice. This is consistent with previous observations that the presence of $t$-butyl chloride, carbon tetrachloride or hexamethyldisilane $(<10 \%)$ in the hexamethylethane lattice increases the rate of self-diffusion of the hexamethylethane molecules and decreases the melting point. ${ }^{3}$ Indeed, melting appears to occur when the solid-state diffusion reaches a certain critical value. It is reasonable to assume that molecules which diffuse quickly in the host enhance the diffusion rate of the latter and vice versa.

The methyl proton signal is much wider than the carboxyl proton signal in neat PA, whereas this difference is small or negligible for the binary samples. A molecule of neat PA forms dimers with one or another of its twelve nearest neighbours of the lattice, fluctuating among them by means of the tumbling motion which is much faster than the molecular self-diffusion. ${ }^{5,17}$ It follows that rapid exchange of the acid protons within the dimeric unit, in combination with reorientation of individual monomers, provides a mechanism by which the carboxyl hydrogens can move through the lattice at a much faster rate than the rest of the molecule. If this model is correct, the activation energy of the acid hydrogen diffusion $\left(18 \mathrm{~kJ} \mathrm{~mol}^{-1}\right.$ for neat PA) reflects the reorientation of individual monomers. In the binary samples one or more of the nearest neighbours of a given PA molecule is replaced by $\mathrm{CCl}_{4}$ molecules. The rate of migration of the acid protons in the lattice is thus reduced, thereby making this linenarrowing mechanism less effective in comparison with whole-molecule diffusion. In fact, at intermediate concentrations of PA $\left(X_{\mathrm{PA}}=0.5-0.7\right)$ the line-narrowing data of the methyl and carboxyl protons provide identical activation energies (Fig. 4), showing that whole-molecule diffusion dominates the line-narrowing mechanism in both cases. When the PA concentration is further reduced, the $E_{\mathrm{d}}$ value for the $\mathrm{COO}^{1} \mathrm{H}$ group falls below that for the methyl group. This shows that the relative rate of migration of the acid protons is again increasing.

Spin-lattice relaxation. The $T_{1}$ values of the binary samples were measured for the liquid $\left({ }^{1} \mathrm{H},{ }^{2} \mathrm{H}\right.$ and $\left.{ }^{17} \mathrm{O}\right)$, disordered $\left({ }^{1} \mathrm{H}\right.$ and $\left.{ }^{2} \mathrm{H}\right)$ and ordered $\left({ }^{1} \mathrm{H}\right)$ phases. Labelled pivalic acid (PA-d) was used when measuring $T_{1}$ of the carboxylic deuteron and oxygen-17 in the mixtures, whereas unlabelled PA was used for all the other measurements. The $T_{1}$ data reflect the motion of the PA (or PA-d) molecules alone. Semi-logaritmic plots of $T_{1}$ versus inverse temperature are shown in Figs. 5 and 6. Some hysteresis is seen in the warming/cooling data near 
the melting point due to undercooling or superheating. Apart from that, the thermal history of the sample, in particular the rate of cooling or warming, appears to have no noticeable effect on $T_{1}$.

Molecular self-diffusion is too slow to modulate the intermolecular spin-lattice relaxation of the PA molecules. Dilution in $\mathrm{CCl}_{4}$ will, in any case, make the intermolecular contribution negligible. The prime source of ${ }^{1} \mathrm{H} T_{1}$ relaxation of the methyl protons therefore arises from intramoleculer dipole-dipole interactions modulated by overall molecular tumbling and/or internal rotations of the methyl and $t$-butyl groups $\left(C_{3}\right.$ and $C_{3}^{\prime}$ motions, respectively). The relaxation of the $\mathrm{CH}_{2}^{2} \mathrm{H}$ and $\mathrm{COO}^{2} \mathrm{H}$ deuterons and ${ }^{17} \mathrm{O}$ nuclei is, on the other hand, governed by the quadrupolar relaxation mechanism.

Ordered solid. In this phase only the internal $C_{3}$ and $C_{3}^{\prime}$ reorientations with correlation times $t_{\mathrm{m}}$ and $\tau_{\mathrm{M}}$, respectively, modulate the dipolar ${ }^{1} \mathrm{H} T_{1}$ relaxation. It is readily shown that ${ }^{5,18}$ eqn. (1) holds, where $\tau_{1}=\tau_{M}, \tau_{2}=\tau_{\mathrm{m}}$

$$
\begin{aligned}
\frac{1}{T_{1}\left({ }^{1} \mathrm{H}\right)}= & \left(\frac{\mu_{\mathrm{O}}}{4 \pi}\right)^{2} \frac{7}{12} \gamma_{\mathrm{H}}^{4} \hbar^{2} \\
& \times\left[r_{\mathrm{HH}}^{-6} \sum_{i=1}^{3} A_{i} g\left(\omega_{\mathrm{H}}, \tau_{i}\right)+A_{4} r_{\mathrm{HH}^{\prime}}^{-6} g\left(\omega_{\mathrm{H}}, \tau_{\mathrm{M}}\right)\right]
\end{aligned}
$$

and $\tau_{3}^{-1}=\tau_{\mathrm{M}}^{-1}+\tau_{\mathrm{m}}^{-1}$, and the spectral density function is given by eqn. (2).

$g\left(\omega_{\mathrm{H}}, \tau_{i}\right)=\frac{\tau_{i}}{1+\omega_{\mathrm{H}}^{2} \tau_{i}^{2}}+\frac{4 \tau_{i}}{1+4 \omega_{\mathrm{H}}^{2} \tau_{i}^{2}}$

If all carbon atoms of the $t$-butyl group have tetrahedral geometry, $A_{1}=A_{2}=8 / 35, A_{3}=19 / 35$ and $A_{4}=81 / 35$. In the following calculations the $\mathrm{C}-\mathrm{H}$ and $\mathrm{C}-\mathrm{C}$ bond lengths are assumed to be 0.110 and $0.154 \mathrm{~nm}$, respectively, giving $r_{\mathrm{HH}}=0.180 \mathrm{~nm}$ and $r_{\mathrm{HH}^{\prime}}=0.311 \mathrm{~nm}$.

Eqn. (1) predicts two minima when the correlation times $\tau_{m}$ and $\tau_{M}$ become very different, as observed for $t$-butyl alcohol ${ }^{19}$ and $t$-butyl cyanide. ${ }^{20}$ The single but slightly asymmetric $T_{1}$ minimum observed for neat PA and the most concentrated binary samples (Fig. 5) suggests that the two types of motion have comparable correlation times but somewhat different $E_{\mathrm{a}}$ values. We believe, in accordance with previous observations, ${ }^{5}$ that the $C_{3}^{\prime}$ and $C_{3}$ reorientations dominate the $T_{1}$ relaxation on the low- and high-temperature regions of the $T_{1}$ curve, respectively.

Two striking features distinguish the $T_{1}$ curves for the binary samples below their transition temperatures (Fig. 5). Firstly, the $T_{1}$ curves become significantly broader on increasing dilution. Secondly, the value of $T_{1}$ at the minimum $\left(T_{1 \min }\right)$ increases from $149 \mathrm{~ms}$ in the pure material to $221-353 \mathrm{~ms}$ in the diluted samples. These observations suggest that two distinct correlation times, $\tau_{\mathrm{m}}$ and $\tau_{\mathrm{M}}$, are inadequate to describe the relaxation time data of the binary samples. Indeed, the behaviour seen above is characteristic of systems whose $T_{1}$ relaxation is described by a distribution of correlation times. ${ }^{3}$ Such a distribution could result from non-uniform environments seen by the various methyl groups. Generally, this would result in a more shallow minimum and an increase in the value of $T_{1 \text { min }}$ relative to that expected for a process governed by a single correlation time. This effect arises since not all the motions are equally effective in causing relaxation at any given temperature.

The position of the $T_{1}$ minimum moves to lower temperature with increasing dilution. This observation suggests that the reorientational freedom of the $t$-butyl groups is enhanced by the addition of $\mathrm{CCl}_{4}$. This is reasonable, since the $C_{3}^{\prime}$ rotation, which is believed to dominate the $T_{1}$ minimum, is largely of intermolecular origin. The increase in the rate of reorientation of the PA molecules is accompanied by a decrease in the transition temperature (Fig. 2).

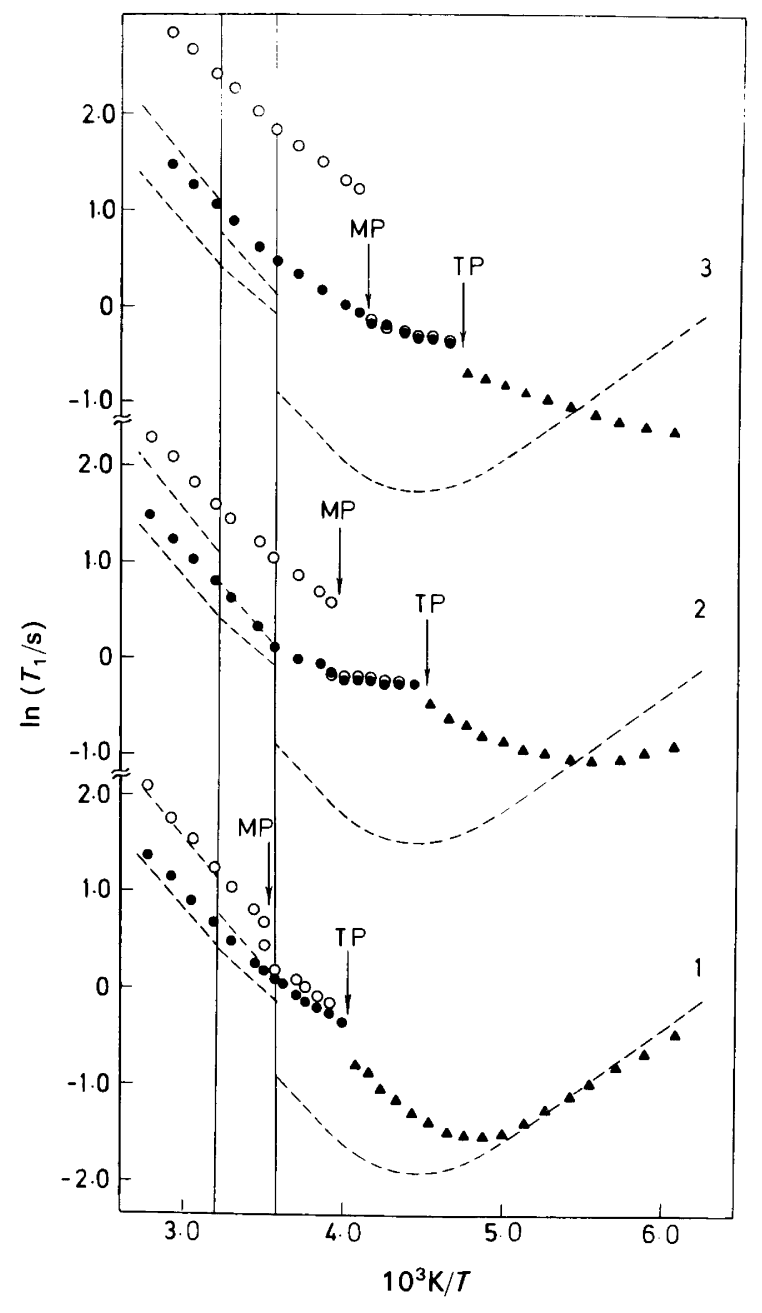

Fig. 5. Proton $T$, relaxation times of binary mixtures of PA and $\mathrm{CCl}_{4}$ versus reciprocal temperature at $0.84(1), 0.47$ (2) and 0.06 (3) mole fractions of PA: $\mathrm{O}, \mathrm{COOH}$ proton; $\bullet, \mathrm{CH}_{3}$ protons; $\boldsymbol{\Lambda}$, all protons. The dashed lines show the corresponding $T_{1}$ values for neat PA. 


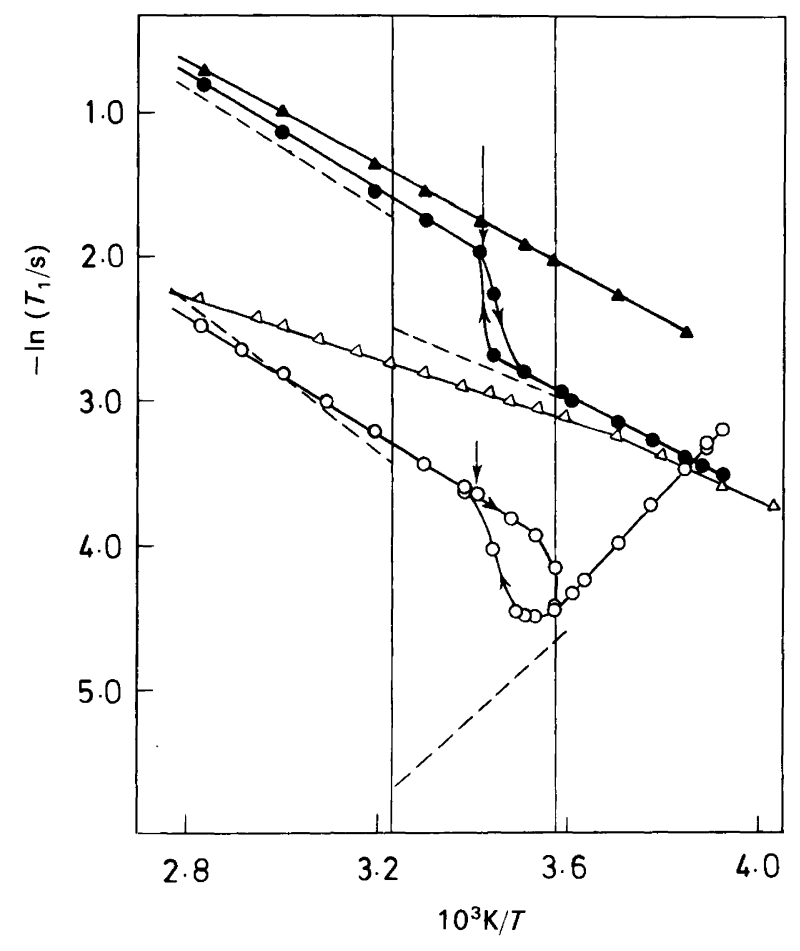

Fig. 6. Deuteron $T_{1}$ relaxation times of binary mixtures of PA and $\mathrm{CCl}_{4}$ versus reciprocal temperature: $\bullet$ and $\Delta, \mathrm{CH}_{2}{ }^{2} \mathrm{H}$ at a mole fraction of 0.86 and 0.39 , respectively; $O$ and $\triangle$, $\mathrm{COO}^{2} \mathrm{H}$ at a mole fraction of 0.89 and 0.09 , respectively. The dashed lines show the corresponding $T_{1}$ values for neat PA.

Liquid and disordered phases. The negative slopes of the ${ }^{1} \mathrm{H},{ }^{2} \mathrm{H}$ and ${ }^{17} \mathrm{O}$ (not shown) $T_{1}$ curves in the liquid and partly the disordered phases (Figs. 5 and 6) imply that the extreme narrowing condition prevails. The relevant ${ }^{1} \mathrm{H}$ and ${ }^{2} \mathrm{H}$ relaxation rates are therefore given by eqns. (3) and $(4),{ }^{5}$ where $\mathrm{X}=\mathrm{C}$ or $\mathrm{O}, \tau_{\mathrm{eff}}$ is the effective correlation

$\frac{1}{T_{1}\left({ }^{1} \mathrm{H}\right)}=\left(\frac{\mu_{\mathrm{O}}}{4 \pi}\right)^{2} 3 \gamma_{\mathrm{H}}^{4} \hbar^{2}\left(r_{\mathrm{HH}}^{-6} \tau_{\mathrm{eff}}^{\mathrm{HH}}+3 \tau_{\mathrm{HH}^{\prime}}^{-6} \tau_{\mathrm{eff}}^{\mathrm{HH}^{\prime}}\right)$

$\frac{1}{T_{1}\left({ }^{2} \mathrm{H}\right)}=\frac{3}{2} \pi^{2} \chi^{2} \tau_{\mathrm{eff}}^{\mathrm{XH}}$

time, $r_{\mathrm{HH}}$ is the internuclear $\mathrm{H} \cdots \mathrm{H}$ separation of a $\mathrm{CH}_{3}$ group, $r_{\mathrm{HH}^{\prime}}$ is the distance between the centres of the equilateral triangles formed by the three hydrogen atoms of each methyl group and $\chi=e^{2} q Q / h$ is the quadrupolar coupling constant. The $\tau_{\text {eff }}$ terms in eqns. (3) and (4) are functions of the correlation times of the contributing motions. In the calculations we used the $\chi$ values reported for neat PA, ${ }^{5}$ viz. 173 and $175 \mathrm{kHz}$ for the methyl and hydroxyl deuterons, respectively.

Since the principal field gradient at the $\mathrm{COO}^{2} \mathrm{H}$ deuteron is almost parallel with the long axis of the dimer, the ${ }^{2} \mathrm{H}$ relaxation will not be affected by a possible rotation about this axis. The $T_{1}$ data of the $\mathrm{COO}^{2} \mathrm{H}$ deuteron therefore reflect the overall tumbling motion alone with

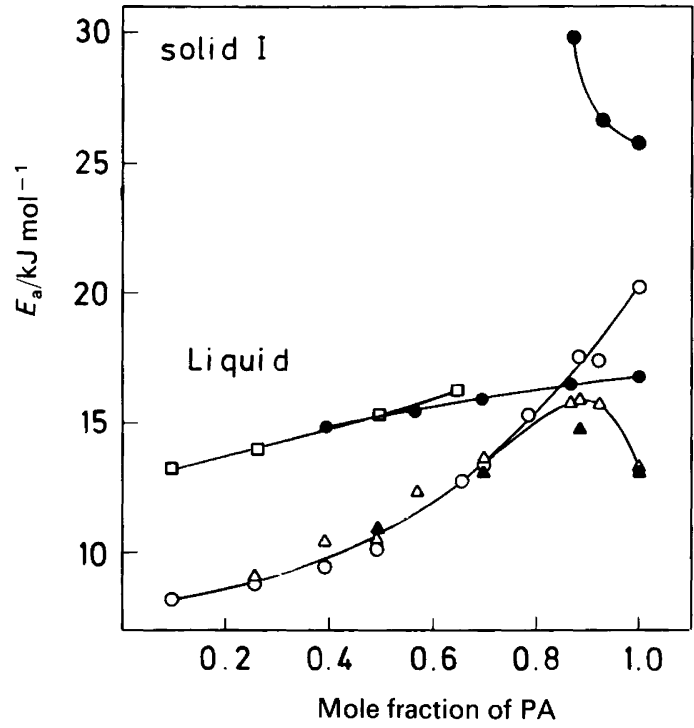

Fig. 7. Activation energies obtained from $T_{1}$ and linewidth measurements as a function of composition in the liquid and disordered phases of the binary system PA-CCl $\mathrm{Cl}_{4}$. Circles: $T_{1}$ of the $\mathrm{COO}^{2} \mathrm{H}(\mathrm{O})$ and $\mathrm{CH}_{2}{ }^{2} \mathrm{H}(\bullet)$ deuterons above $280 \mathrm{~K}$; squares: $T_{1}$ of the $\mathrm{COO}^{2} \mathrm{H}$ deuteron $(\square)$ below $280 \mathrm{~K}$; triangles: linewidth $(\Delta)$ and $T_{1}(\Delta)$ of the carboxyl oxygen-17.

correlation time $\tau_{\mathrm{r}}$, which is directly obtainable from eqn. (5) by replacing $\tau_{\text {eff }}^{\mathrm{XH}}$ by $\tau_{\mathrm{r}}$. The $T_{1}$ curves of diluted PA show an abrupt change of slope and concomitant activation energy, around $280 \mathrm{~K}$ (Figs. 6 and 7). Similar observations have been made for the corresponding linewidths and chemical shifts. ${ }^{21}$ The most reasonable explanation of these singularities is a second-order phase transition in the ensemble of cyclic dimers. ${ }^{21}$ Below a certain temperature $T_{\mathrm{c}}$ (ca. $280 \mathrm{~K}$ ) a lowering of the average symmetry of the cyclic dimers, from a $D_{2 \mathrm{~h}}$ structure above $T_{\mathrm{c}}$ to a $C_{2 \mathrm{~h}}$ structure below $T_{\mathrm{c}}$, may take place due to a slowing down of the proton exchange motion. An alternative possibility, namely formation of larger aggregates, could also explain the peculiar temperature behaviour of the NMR parameters. In the latter case $T_{\mathrm{c}}$ could be interpreted as a critical temperature above which the breaking of these aggregates occurs.

The activation energy $\left(E_{\mathrm{a}}\right)$ for the overall tumbling motion $\left(\tau_{r}\right)$ above $280 \mathrm{~K}$ in the liquid state decreases monotonically from $20.2 \mathrm{~kJ} \mathrm{~mol}^{-1}$ in neat PA to $8.6 \mathrm{~kJ} \mathrm{~mol}^{-1}$ in the most diluted sample $\left(X_{\mathrm{PA}}=0.09\right)$ (Fig. 7). The corresponding correlation time undergoes a similar reduction from 63 to 34 ps at $310 \mathrm{~K}$ (the melting point of neat PA). These observations are largely attributed to reduced viscosity and steric hindrance. The apparent activation energy of the methyl group is not reduced to the same extent on dilution (Fig. 7). This is reasonable, since $E_{\text {a }}$ here mainly reflects internal motions which are largely controlled by intramolecular interactions. Fig. 7 also shows activation energies obtained from the oxygen-17 linewidth and $T_{1}$ data in the liquid phase. The $E_{\mathrm{a}}$ values agree well with those obtained from the corresponding deuteron measurements except 
for the neat PA sample. A certain deviation is expected, however, since the average ${ }^{17} \mathrm{O}$ field gradient will, unlike the situation for the $\mathrm{COO}^{2} \mathrm{H}$ deuteron, not be parallel with the long axis of the dimer.

$T_{1}\left({ }^{2} \mathrm{H}\right)$ of the disordered phase was only observed for the more concentrated samples and neat PA ( $\left.X_{\mathrm{PA}}=0.89-1\right)$ owing to increasing broadening and reduced signal (Fig. 6). The positive slopes of the $T_{1}$ curves of the disordered phase imply that the relaxation is outside the extreme narrowing regime, i.e. $\tau_{\mathrm{r}}>1.6 \mathrm{~ns}$. Considerable steric hindrance against overall molecular tumbling is reflected in the high activation energy $\left(26-30 \mathrm{~kJ} \mathrm{~mol}^{-1}\right)$ and long correlation times (>1.6 ns) observed for these samples (Fig. 7). It is well known that molecules do not have sufficient space to rotate freely in the disordered phase, and change of orientation therefore requires a correlated movement of neighbouring molecules. Even a small fractional concentration of $\mathrm{CCl}_{4}$ molecules in the lattice $(\leqslant 11 \%)$ is seen to produce a significant effect on the overall tumbling motion, apparently by progressively changing the dimension of the unit cell.

\section{References}

1. See, e.g., Sherwood, J. N. The Plastically Crystalline State, Wiley, New York 1979, and references therein.

2. See, e.g., Parsonage, N. G. and Staveley, L. A. K. Disorder in Crystals, Clarendon Press, Oxford 1978, pp. 680-690, and references therein.
3. Britcher, A. R. and Strange, J. H. J. Chem. Phys. 75 (1981) 2029.

4. Akimov, M. N., Bezrukov, O. F., Chikunov, O. V. and Struts, A. V. J. Chem. Phys. 95 (1991) 22.

5. See, e.g. Aksnes, D. W. and Kimtys, L. L. Magn. Reson. Chem. 28 (1990) S20, and references therein.

6. Kondo, S. and Oda, T. Bull. Chem. Soc. Jpn. 27 (1954) 567.

7. Longueville, W., Fontaine, H., Baert, F. and Odou, G. Acta Crystallogr., Sect. A 34 (1978) S188.

8. Suga, H., Sugisaki, M. and Seki, S. Mol. Cryst. 1 (1966) 377.

9. Namba, H. and Oda, T. Bull. Chem. Soc. Jpn. 25 (1952) 225.

10. Longueville, W. and Fontaine, H. Mol. Cryst. Liq. Cryst. 32 (1976) 73.

11. Rudman, R. and Post, B. Science 154 (1966) 1009.

12. Rudman, R. and Post, B. Mol. Cryst. 5 (1968) 95.

13. Kotake, K., Nakamura, N. and Chihara, H. Bull. Chem. Soc. Jpn. 40 (1967) 1018.

14. Silver, L. and Rudman, R. J. Phys. Chem. 74 (1970) 3134.

15. Vold, R. L., Waugh, J. S., Klein, M. P. and Phelps, D. E. J. Chem. Phys. 48 (1968) 3831.

16. Corke, N. T., Lockhart, N. C., Narang, R. S. and Sherwood, J. N., Mol. Cryst. Liq. Cryst. 44 (1978) 45.

17. Jackson, R. L. and Strange, J. H. Mol. Phys. 22 (1971) 313.

18. Albert, S., Gutowski, H. S. and Ripmeester, J. A. J. Chem. Phys. 64 (1976) 3277.

19. Aksnes, D. W. and Kimtys, L. L. Magn. Reson. Chem. 29 (1991) 698 .

20. Aksnes, D. W., Kimtys, L. L. and Ramstad. K. Magn. Reson. Chem. 30 (1992) 865.

21. Kimtys, L., Balevicius, V. and Aksnes, D. W. J. Mol. Struct. 270 (1992) 161.

Received June 5, 1992. 